\title{
Effect of 'rice' pattern on high blood pressure by gender and obesity: using the community-based KoGES cohort
}

\author{
Yuri Han ${ }^{1}$, Daehee Kang ${ }^{2}$ and Sang-Ah Lee ${ }^{1,3, *}$ \\ 'Department of Preventive Medicine, Kangwon National University School of Medicine, Chuncheon-si, Gangwon-do \\ 24341, Republic of Korea: 'Department of Preventive Medicine, College of Medicine, Seoul National University, \\ Seoul, Republic of Korea: ${ }^{3}$ Division of Epidemiology, Vanderbilt University Medical Center, Nashville, TN 37203, \\ USA
}

Submitted 26 October 2018: Final revision received 25 February 2019: Accepted 5 April 2019: First published online 8 July 2019

\begin{abstract}
Objective: The present study aimed to examine the association between dietary pattern and the risk of high blood pressure (BP) and to estimate the attenuated effect by gender and obesity on the association using data from a prospective cohort study in Korea.

Design: Prospective study. Diet was assessed using a validated 103-item FFQ and was input into factor analysis after adjustment for total energy intake.

Setting: Community-based Korean Genome Epidemiology Study (KoGES) cohort. Participants: Healthy individuals ( $n$ 5151) without high BP at recruitment from the community-based cohort study.

Results: Dietary pattern was not associated with the risk of high BP regardless of the type of covariates, with the exception of the 'rice' pattern. The effect of the 'rice' pattern was observed in both men $\left(P_{\text {trend }}=0.013\right)$ and women $\left(P_{\text {trend }}<0.001\right)$, but the statistical significance remained only in women after adjustment for confounders $\left(P_{\text {trend }}=0.004\right)$. The positive association of the 'rice' pattern with high BP risk was attenuated by obesity. After stratification by gender and obese status, in particular, the harmful effect of the 'rice' pattern was predominantly observed in obese women $\left(P_{\text {trend }}<0 \cdot 001\right)$ only.

Conclusions: This longitudinal study in Korean adults found a positive association of the 'rice' pattern with long-term development of incident high BP, predominantly in women. The association is likely to be attenuated by gender and obese status.
\end{abstract}

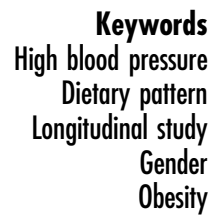

The prevalence of raised blood pressure (BP) is $22 \%$ in adults aged 18 years or above worldwide ${ }^{(1)}$, and its harmful effect on CVD and related mortality has already been supported by large prospective epidemiological studies ${ }^{(2)}$. According to the Joint National Committee's 7 th report $^{(3)}$, a healthy lifestyle needs to be adopted for the prevention and management of $\mathrm{BP}$, including weight reduction, consumption of foods rich in $\mathrm{K}$ and $\mathrm{Ca}$, dietary $\mathrm{Na}$ reduction, physical activity and modification of alcohol consumption. The Dietary Approaches to Stop Hypertension (DASH) diet plan is the most popular to reduce the prevalence of high BP.

Several meta-analyses ${ }^{(4,5)}$ and a large cross-sectional study (INTERMAP) ${ }^{(6)}$ have reported the association between intake of single nutrients and $\mathrm{BP}$, but the results are not consistent. Because people consume complex combinations of foods, it is difficult to assess the independent effects of single nutrients or foods. Therefore, dietary pattern analysis has been performed recently to evaluate the effect of food/nutrient intake on chronic diseases including high $\mathrm{BP}^{(7)}$. Although the adaptation of the DASH diet plan for reducing BP has been supported by several prospective studies and intervention trials, its beneficial effect remains controversial. In particular, the traditional dietary items and cooking methods of Asian populations were shown to result in more complicated and varied relationships between dietary factors and BP compared with those in Western populations ${ }^{(8-14)}$.

Although the main issue of dietary factors to prevent high BP has traditionally focused on fat intake, especially saturated fat, dietary carbohydrate intake may play a substantial role in the risk of high BP for Asians, because they traditionally consume large amounts of rice as a staple food. The effects of a carbohydrate-rich diet and high

*Corresponding author: Email sangahlee@kangwon.ac.kr

(C) The Authors 2019. This is an Open Access article, distributed under the terms of the Creative Commons Attribution-NonCommercialNoDerivatives licence (http://creativecommons.org/licenses/by-nc-nd/4.0/), which permits non-commercial re-use, distribution, and reproduction in any medium, provided the original work is unaltered and is properly cited. The written permission of Cambridge University Press must be obtained for commercial re-use or in order to create a derivative work 
refined-grain consumption on the risk of high $\mathrm{BP}$ have been observed in obese Korean women ${ }^{(15)}$ and South Indians $^{(16)}$, respectively. Clinical trials using polyunsaturated fat to replace saturated fat have reduced the incidence of CVD, but use of carbohydrates to replace saturated fat did not decrease the incidence ${ }^{(17,18)}$. On the other hand, evidence from prospective observational studies indicates that carbohydrates from whole grains could reduce CVD when they replace saturated fat ${ }^{(19)}$.

Regarding the effect of diet on the risk of high BP by ethnic variation, it is valuable to consider the attenuated effect by aetiological characteristics including gender and obesity $^{(20,21)}$. Therefore, the aim of the present study was to examine the association between dietary pattern and the risk of high BP and to evaluate the attenuated effect on the association by gender and obesity using a prospective cohort study.

\section{Methods}

\section{Study population}

Data from participants in the community-based Korean Genome Epidemiology Study (KoGES), which is a cohort study in Korea, were used to investigate the effect of dietary and environmental factors on chronic diseases. Detailed information on the study procedure has been described previously $^{(22)}$. Briefly, 10038 (urban area, $n$ 5020; rural area, $n$ 5018) participants were recruited from 2001 to 2003 for baseline examinations. Follow-up examinations were then performed every 2 years.

Detailed information on the selection of participants for the current study is presented in Fig. 1. Among these 10038 participants, we excluded thirty-nine participants who were under 40 or over 70 years of age at baseline recruitment and those for whom no information was available on BP ( $n 16)$, history of hypertension ( $n 4)$ or total daily energy intake $(n 318)$. As well as those who had a history of chronic disease (including hypertension and hypertension medication use, diabetes mellitus, hyperlipidaemia, CVD and cancer; $n$ 2289), participants were also excluded who reported implausible total daily energy intake ( $\leq 3357$ or $\geq 16736 \mathrm{~kJ}$ ( $\leq 800$ or $\geq 4000 \mathrm{kcal}$ ) for males, $\leq 2092$ or $\geq 14644 \mathrm{~kJ}$ ( $\leq 500$ or $\geq 3500 \mathrm{kcal})$ for females; $n$ 208). Participants who had been diagnosed with high BP at baseline ( $n$ 1383) were excluded to eliminate a latent period bias. After exclusions, 5781 individuals were included as our study population. Of these, 630 did not participate in any follow-up through the first to the fourth after the baseline examination. Finally, 5151 healthy individuals were analysed.

\section{Definition of blood pressure incidence}

BP measurements were performed at each follow-up examination. Systolic BP (mmHg) and diastolic BP
$(\mathrm{mmHg})$ were measured repeatedly in both arms while the participant was in a sitting posture. The mean value of repeated measurements was used to determine high $\mathrm{BP}$, defined as systolic $\mathrm{BP} \geq 140 \mathrm{mmHg}$ or diastolic $\mathrm{BP} \geq 90 \mathrm{mmHg}$ according to the WHO criteria.

To define the incidence of high BP, we took cases who were first diagnosed with high BP through the first to the fourth follow-up. The detailed process to define event (high BP) and non-event (normal BP) participants is presented in Fig. 1.

\section{Socio-economic and lifestyle factors}

To analyse factors affecting the risk of high BP, socioeconomic and lifestyle factors were surveyed. General factors included age, gender, education level (<elementary, middle school, high school, $\geq$ college), monthly average household income in US dollars $(<1000,1000-1999$, 2000-3999, $\geq 4000$ ), occupation (office, non-office, housework), marital status (yes/no), family history of hypertension (yes/no), BMI in kg/m² (<23, 23-24.9, 25-29.9, $\geq 30)$ and waist circumference (males, $<90 \mathrm{~cm}$; females, $<85 \mathrm{~cm}$ ). Lifestyle factors included drinking status (noncurrent $v$. current), total alcohol consumption (g/d), smoking status (non-current $v$. current), absolute number of cigarettes smoked (packs/year), physical activity (yes/ no) and total energy intake (quartiles).

\section{Dietary intake assessment}

Dietary intake information was measured using the semiquantitative FFQ developed for KoGES ${ }^{(23)}$. This FFQ consisted of 103 items concerning the individual's food intake over the past year. The frequency of consumption for each item was divided into nine categories: 'never or seldom', 'once a month', '2-3 times a month', '1-2 times a week', '3-4 times a week', '5-6 times a week', 'once a day', 'twice a day' and 'three times or more a day'. For a semi-quantitative approach, one serving size was classified into less ( 0.5 times), reference amount and higher (1.5 times). The duration of seasonal variety of fruit intake was divided into four categories (3, 6, 9 and 12 months). Validity of the developed FFQ was then conducted. The correlation coefficient for nutrients ranged from 0.23 (vitamin A) to 0.64 (carbohydrate).

\section{Dietary pattern derivation}

Factor analysis was used to derive dietary patterns and determine factor loadings for daily intake of 103 individual food items. These factors were rotated with varimax rotation to maintain uncorrelated factors and enhance interpretability. We examined both scree plots and factors to determine which set of factors most closely described food consumption patterns after adjusting for total energy intake. The factor score for each pattern was calculated by summing the intake of all food groups weighted by their factor loadings. A factor score was then calculated for each 


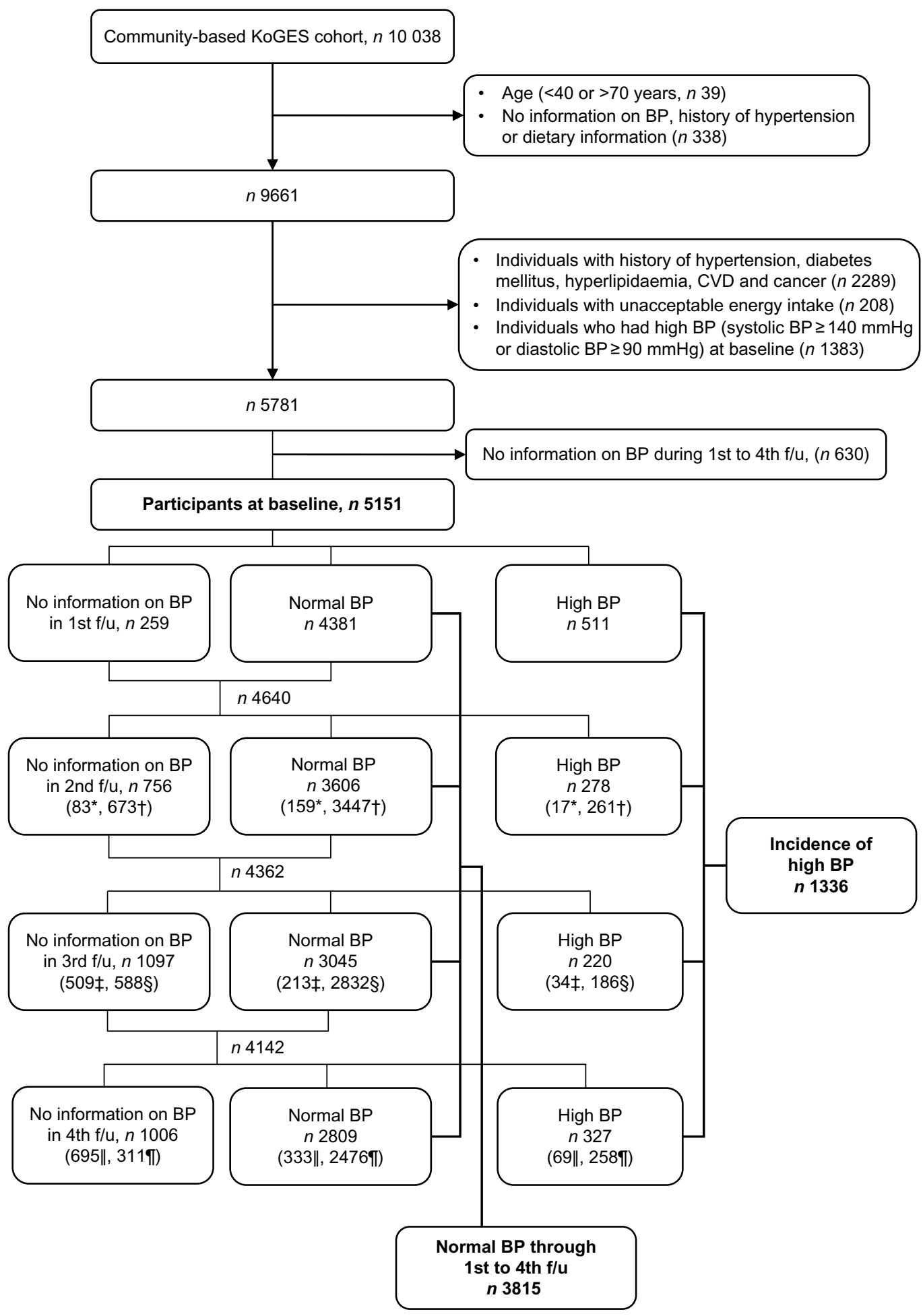

Fig. 1 Flow diagram showing the selection of participants for the present study. *From individuals who had not participated in the 1 st $f / u$. †From individuals who had participated in the $1 \mathrm{st} f / \mathrm{u}$. \#From individuals who had not participated in the 2 nd f/u. §From individuals who had participated in the 2 nd f/u. $\|$ From individuals who had not participated in the $3 \mathrm{rd} f / \mathrm{u}$. ๆFrom individuals who had participated in the 3rd f/u (KoGES, Korean Genome Epidemiology Study; BP, blood pressure; f/u, follow-up) 
participant for each of the four factors, in which standardized intake for each of the 103 foods was weighted by its factor loadings and summed. From these analyses, five dietary patterns with the highest loadings (absolute loading $>0.30$ ) were identified and named according to the highest loading food. The following dietary patterns were defined: (i) 'fruit' pattern with high fruit score; (ii) 'vegetable' pattern with high score for oyster mushroom, onion, other mushroom, perilla leaf, leek/parsley, spinach and courgette; (iii) 'high-protein' pattern with high score for sashimi (flounder, crab, tuna, urogu, etc.), pork roast (ribs, sirloin, etc.), grits, roast beef, pork belly and eel; (iv) 'coffee' pattern with high score for sugar, coffee and coffee creamer; and (v) 'rice' pattern with low score for unrefined cereal but high score for refined rice, nabak kimchi/ dongchimi and kakdugi (see online supplementary material, Supplemental Table S1).

\section{Statistical analysis}

General and lifestyle factors according to each dietary pattern score were presented using frequency or mean and SD. Pearson correlation analysis was performed to examine the correlation between each dietary pattern and nutrients. To examine the effect of each dietary pattern on the risk of high BP, the Cox proportional hazards model was performed after adjustment for covariates that were associated with high BP in this study population; including age, gender, education level, monthly average household income, family history of hypertension, BMI, waist circumference and total energy intake. To evaluate the attenuated effect of obesity and gender on the association between the 'rice' pattern and high BP risk, we analysed the association between the 'rice' pattern and high BP risk after stratifying by obesity (BMI $<25 \mathrm{~kg} / \mathrm{m}^{2} v$. BMI $\geq 25 \mathrm{~kg} / \mathrm{m}^{2}$ ) for both genders. All analyses were performed using the statistical software package SAS version 9.3. Statistical significance was considered at $P<0.05$.

\section{Results}

\section{Characteristics of dietary patterns}

The characteristics of participants for each dietary pattern are shown in Table 1. Elderly participants were more likely to belong in the group of individuals with high 'fruit' and 'rice' pattern scores but with low 'high-protein' and 'coffee' pattern scores. Men were likely to rank high for the 'highprotein', 'coffee' and 'rice' patterns. However, women had high scores for the 'fruit' and 'vegetable' patterns. Participants who had an education level of more than high school and those who reported relatively high family income (more than \$US 2000/month) had high scores for the 'high-protein' pattern, but low scores for the 'rice' pattern. Office workers had high scores for the 'high-protein' and 'coffee' patterns, while blue-collar workers had high scores for the 'rice' pattern. Participants with a family history of hypertension had higher scores for the 'highprotein' pattern but lower scores for the 'rice' pattern compared with those without such history. No significant difference was observed among scores for the different dietary patterns according to BMI, but abdominal obesity was observed in participants with high scores for the 'fruit' and 'rice' patterns but low scores for the 'high-protein' pattern. Current drinkers had high scores for the 'high-protein' pattern but low scores for the 'fruit' pattern. Higher scores for the 'high-protein', 'coffee' and 'rice' patterns but lower scores for the 'fruit' and 'vegetable' patterns were observed in current smokers compared with those in non-smokers. Current exercisers were likely to have low scores for the 'rice' pattern.

\section{Correlations between nutrients and dietary patterns}

The results of correlation coefficients between nutrients and each dietary pattern are presented in Table 2 . The 'fruit' pattern showed a high correlation with vitamin C $(r=0.68)$, $\mathrm{K}(r=0 \cdot 30)$ and fibre $(r=0 \cdot 26)$. The 'vegetable' pattern was correlated with overall vitamins (ranged from $r=0.25$ for niacin to $r=0.55$ for vitamin A and folate) and minerals (ranged from $r=0.31$ for $\mathrm{P}$ to $r=0.49$ for $\mathrm{Fe}$ ). The 'highprotein' pattern had positive correlations with protein, fat, retinol, niacin and cholesterol levels. However, the 'coffee' pattern showed no correlation with any nutrient. The 'rice' pattern also had no correlation with any nutrient, with the exception of $\mathrm{Na}(r=0 \cdot 28)$.

\section{Association of ecological and lifestyle factors with the risk of high $B P$}

The average follow-up time was $6 \cdot 2$ years and ranged from 1.4 to 8.7 years. The risk of high BP increased with age $\left(P_{\text {trend }}<0.001\right)$ and in women (hazard ratio $=0.65,95 \%$ CI $0.57,0.76$; Table 3). Education level $\left(P_{\text {trend }}<0.001\right)$ and family income $\left(P_{\text {trend }}<0.001\right)$ were negatively associated with high BP risk. Family history of hypertension showed increased risk of high BP (hazard ratio $=1.50$, $95 \%$ CI $1.30,1.73)$. Both general obesity $\left(P_{\text {trend }}<0.001\right)$ and abdominal obesity (hazard ratio $=1.54,95 \%$ CI 1.33 , 1.77) were associated with increased risk of high BP. However, lifestyle factors such as drinking, smoking, exercise and total energy intake showed no significant association with the risk of high BP.

\section{Effect of dietary patterns on the risk of high blood pressure}

No association was shown for each dietary pattern with the risk of high BP regardless of the type of covariates, with the exception of the 'rice' pattern (Table 4). The effect of the 'rice' pattern was observed in both men $\left(P_{\text {trend }}=0.013\right)$ and women $\left(P_{\text {trend }}<0.001\right)$ after adjusting for age. After adjusting for all confounding factors, its statistical 
Table 1 Characteristics of participants in each dietary pattern. Korean adults ( $n 5151)$ from the community-based KoGES cohort

\begin{tabular}{|c|c|c|c|c|c|c|c|c|c|c|c|c|c|c|c|c|c|c|c|c|}
\hline & \multicolumn{4}{|c|}{ 'Fruit' pattern } & \multicolumn{4}{|c|}{ 'Vegetable' pattern } & \multicolumn{4}{|c|}{ 'High-protein' pattern } & \multicolumn{4}{|c|}{ 'Coffee' pattern } & \multicolumn{4}{|c|}{ 'Rice' pattern } \\
\hline & \multicolumn{2}{|c|}{ Q1 } & \multicolumn{2}{|c|}{ Q5 } & \multicolumn{2}{|c|}{ Q1 } & \multicolumn{2}{|c|}{ Q5 } & \multicolumn{2}{|c|}{ Q1 } & \multicolumn{2}{|c|}{ Q5 } & \multicolumn{2}{|c|}{ Q1 } & \multicolumn{2}{|c|}{ Q5 } & \multicolumn{2}{|c|}{ Q1 } & \multicolumn{2}{|c|}{ Q5 } \\
\hline & $\begin{array}{l}\text { Mean } \\
\text { or } n\end{array}$ & $\begin{array}{c}\text { SD } \\
\text { or } \%\end{array}$ & $\begin{array}{l}\text { Mean } \\
\text { or } n\end{array}$ & $\begin{array}{c}\text { SD } \\
\text { or } \%\end{array}$ & $\begin{array}{l}\text { Mean } \\
\text { or } n\end{array}$ & $\begin{array}{l}\text { SD } \\
\text { or } \%\end{array}$ & $\begin{array}{l}\text { Mean } \\
\text { or } n\end{array}$ & $\begin{array}{l}\text { SD } \\
\text { or } \%\end{array}$ & $\begin{array}{l}\text { Mean } \\
\text { or } n\end{array}$ & $\begin{array}{l}\text { SD } \\
\text { or } \%\end{array}$ & $\begin{array}{l}\text { Mean } \\
\text { or } n\end{array}$ & $\begin{array}{l}\mathrm{SD} \\
\text { or } \%\end{array}$ & $\begin{array}{l}\text { Mean } \\
\text { or } n\end{array}$ & $\begin{array}{l}\text { SD } \\
\text { or } \%\end{array}$ & $\begin{array}{l}\text { Mean } \\
\text { or } n\end{array}$ & $\begin{array}{l}\text { SD } \\
\text { or } \%\end{array}$ & $\begin{array}{l}\text { Mean } \\
\text { or } n\end{array}$ & $\begin{array}{l}\text { SD } \\
\text { or } \%\end{array}$ & $\begin{array}{l}\text { Mean } \\
\text { or } n\end{array}$ & $\begin{array}{c}\text { SD } \\
\text { or } \%\end{array}$ \\
\hline Age (years) & 49.5 & $8 \cdot 1$ & 51.5 & $8 \cdot 8$ & 50.3 & 8.7 & 50.7 & 8.4 & 54.0 & 8.8 & $47 \cdot 3$ & $6 \cdot 7$ & 51.8 & 8.6 & 49.2 & $8 \cdot 1$ & 47.6 & 6.9 & 53.0 & 8.8 \\
\hline Sex (male) & 683 & $66 \cdot 3$ & 334 & 32.4 & 631 & 61.3 & 364 & $35 \cdot 3$ & 345 & 33.5 & 643 & 62.4 & 430 & 41.7 & 673 & 65.3 & 334 & 32.4 & 628 & 60.9 \\
\hline Education ( $\geq 10$ years) & 582 & $56 \cdot 7$ & 402 & $39 \cdot 3$ & 523 & $51 \cdot 1$ & 474 & $46 \cdot 2$ & 324 & $31 \cdot 6$ & 672 & 65.4 & 443 & 43.3 & 598 & 58.5 & 702 & $68 \cdot 2$ & 324 & 31.6 \\
\hline \multicolumn{21}{|l|}{ Income (\$US/month) } \\
\hline$<1000$ & 256 & 24.8 & 391 & 37.9 & 332 & $32 \cdot 2$ & 318 & 30.9 & 451 & 43.8 & 157 & $15 \cdot 2$ & 344 & 33.4 & 249 & $24 \cdot 2$ & 135 & $13 \cdot 1$ & 470 & 45.5 \\
\hline $1000-1999$ & 310 & $30 \cdot 1$ & 307 & 29.8 & 304 & 29.5 & 296 & 28.7 & 300 & 29.1 & 304 & 29.5 & 317 & $30 \cdot 8$ & 307 & 29.8 & 282 & $27 \cdot 3$ & 315 & 30.5 \\
\hline 2000-3999 & 350 & 34.0 & 248 & $24 \cdot 1$ & 311 & $30 \cdot 2$ & 300 & $29 \cdot 1$ & 222 & 21.6 & 414 & $40 \cdot 2$ & 282 & $27 \cdot 4$ & 363 & $35 \cdot 2$ & 464 & $45 \cdot 0$ & 180 & 17.4 \\
\hline$\geq 4000$ & 103 & 9.99 & $57 \cdot 0$ & 5.53 & $66 \cdot 0$ & $6 \cdot 41$ & 100 & 9.71 & $40 \cdot 0$ & 3.88 & $142 \cdot 0$ & $13 \cdot 8$ & $75 \cdot 0$ & 7.27 & 101 & 9.80 & 145 & $14 \cdot 1$ & 41.0 & 3.97 \\
\hline Blue-collar worker & 728 & $70 \cdot 6$ & 586 & $56 \cdot 8$ & 732 & $71 \cdot 1$ & 564 & 54.8 & 632 & 61.4 & 653 & 63.3 & 630 & $61 \cdot 1$ & 722 & 70.0 & 467 & $45 \cdot 3$ & 797 & $77 \cdot 2$ \\
\hline Married (yes) & 962 & $93 \cdot 3$ & 920 & $89 \cdot 2$ & 949 & $92 \cdot 1$ & 952 & $92 \cdot 4$ & 920 & $89 \cdot 3$ & 972 & $94 \cdot 3$ & 941 & $91 \cdot 3$ & 965 & 93.6 & 964 & 93.4 & 930 & $90 \cdot 1$ \\
\hline $\begin{array}{l}\text { Family history of } \\
\text { hypertension (yes) }\end{array}$ & 169 & $16 \cdot 4$ & 145 & $14 \cdot 1$ & 167 & $16 \cdot 2$ & 157 & $15 \cdot 2$ & 136 & $13 \cdot 2$ & 177 & $17 \cdot 2$ & 138 & 13.4 & 168 & $16 \cdot 3$ & 206 & $20 \cdot 0$ & 121 & $11 \cdot 7$ \\
\hline $\operatorname{BMI}\left(\mathrm{kg} / \mathrm{m}^{2}\right)$ & $24 \cdot 1$ & 2.9 & $24 \cdot 3$ & $3 \cdot 1$ & 23.9 & $3 \cdot 1$ & 24.2 & $3 \cdot 0$ & 24.1 & $3 \cdot 2$ & $24 \cdot 3$ & 2.9 & 23.7 & 2.9 & 24.1 & 3.0 & $24 \cdot 2$ & 3.0 & 23.9 & 3.0 \\
\hline$<23$ & 344 & 33.4 & 365 & 35.4 & 409 & 39.7 & 361 & $35 \cdot 1$ & 393 & 38.2 & 336 & $32 \cdot 6$ & 424 & $41 \cdot 1$ & 364 & $35 \cdot 3$ & 351 & 34.0 & 409 & 39.6 \\
\hline $23-24.9$ & 315 & $30 \cdot 6$ & 273 & 26.5 & 273 & $26 \cdot 5$ & 291 & 28.3 & 257 & $25 \cdot 0$ & 308 & 29.9 & 276 & $26 \cdot 8$ & 301 & $29 \cdot 2$ & 295 & 28.6 & 278 & $26 \cdot 9$ \\
\hline $25-29.9$ & 337 & 32.7 & 347 & 33.7 & 320 & 31.1 & 339 & 32.9 & 331 & $32 \cdot 1$ & 350 & 34.0 & 306 & 29.7 & 329 & 31.9 & 352 & 34.1 & 317 & 30.7 \\
\hline$\geq 30$ & 35.0 & 3.39 & 46.0 & 4.46 & 28.0 & 2.72 & 39.0 & 3.79 & 49.0 & 4.76 & $36 \cdot 0$ & 3.49 & $25 \cdot 0$ & 2.42 & 37.0 & 3.59 & 34.0 & 3.29 & 28.0 & 2.71 \\
\hline Abdominal obesity* & 212 & $20 \cdot 6$ & 297 & 28.9 & 216 & 21.0 & 259 & $25 \cdot 2$ & 281 & $27 \cdot 3$ & 189 & $18 \cdot 4$ & 237 & 23.1 & 194 & 18.9 & 182 & 17.7 & 278 & $27 \cdot 0$ \\
\hline Current drinker & 626 & $60 \cdot 7$ & 391 & 37.9 & 562 & 54.6 & 463 & $45 \cdot 0$ & 384 & $37 \cdot 3$ & 651 & 63.1 & 441 & $42 \cdot 8$ & 570 & $55 \cdot 3$ & 492 & 47.8 & 2505 & 0.0 \\
\hline Current smoker & 384 & $37 \cdot 3$ & 185 & 17.9 & 353 & 34.3 & 217 & $21 \cdot 1$ & 211 & 20.5 & 351 & $34 \cdot 0$ & 175 & $17 \cdot 0$ & 453 & 43.9 & 150 & 14.5 & 401 & 38.9 \\
\hline Regular exerciser & 316 & $30 \cdot 7$ & 247 & 24.0 & 248 & $24 \cdot 1$ & 341 & 33.1 & 247 & 24.0 & 352 & $34 \cdot 1$ & 307 & 29.8 & 241 & 23.4 & 415 & $40 \cdot 3$ & 192 & $18 \cdot 6$ \\
\hline
\end{tabular}

KoGES, Korean Genome Epidemiology Study; Q1, quintile 1; Q5, quintile 5.

Results are presented as mean and SD for age and BMI $\left(\mathrm{kg} / \mathrm{m}^{2}\right)$; and as $n$ and \% for all other variables including BMI groups.

${ }^{*}$ Criteria for abdominal obesity used waist circumference for males and females $\geq 90 \mathrm{~cm}$ and $\geq 85 \mathrm{~cm}$, respectively. 
Table 2 Correlation of nutrients with each dietary pattern after adjusting for total energy intake

\begin{tabular}{lcccrr}
\hline & 'Fruit' pattern & 'Vegetable' pattern & 'High-protein' pattern & 'Coffee' pattern & 'Rice' pattern \\
\hline Protein $(\mathrm{g})$ & -0.02 & 0.22 & 0.26 & -0.03 & -0.11 \\
Fat $(\mathrm{g})$ & -0.07 & 0.04 & 0.35 & 0.07 & -0.15 \\
Sugar $(\mathrm{g})$ & 0.07 & -0.05 & -0.22 & -0.02 & 0.09 \\
Fiber $(\mathrm{g})$ & 0.26 & 0.49 & -0.14 & -0.05 & 0.02 \\
$\mathrm{Ca}(\mathrm{mg})$ & 0.09 & 0.48 & 0.12 & 0 & -0.14 \\
$\mathrm{P}(\mathrm{mg})$ & 0.03 & 0.31 & 0.09 & -0.02 & -0.14 \\
$\mathrm{Fe}(\mathrm{mg})$ & 0.16 & 0.49 & 0.08 & -0.02 & -0.11 \\
$\mathrm{~K}(\mathrm{mg})$ & 0.30 & 0.47 & 0.05 & 0.08 & -0.02 \\
$\mathrm{Na}(\mathrm{mg})$ & -0.02 & 0.41 & 0.03 & 0.04 & 0.28 \\
Zn $(\mathrm{ug})$ & 0 & 0.17 & 0.23 & -0.06 & -0.06 \\
Vitamin A $(\mathrm{RE})$ & 0.10 & 0.55 & 0.15 & 0.01 & 0.10 \\
Retinol $(\mu \mathrm{g})$ & 0.03 & 0.18 & 0.32 & -0.02 & -0.25 \\
Carotene $(\mu \mathrm{g})$ & 0.10 & 0.54 & 0.10 & 0.01 & 0.15 \\
Thiamin $(\mathrm{mg})$ & 0.12 & 0.18 & 0.17 & -0.03 & 0.06 \\
Ribloflavin $(\mathrm{mg})$ & 0.06 & 0.32 & 0.25 & -0.01 & -0.11 \\
Niacin $(\mathrm{mg})$ & 0.06 & 0.25 & 0.29 & 0.11 & -0.08 \\
Vitamin C $(\mathrm{mg})$ & 0.68 & 0.37 & 0.05 & 0.01 & 0.10 \\
Vitamin $\mathrm{B}_{6}(\mathrm{mg})$ & 0.18 & 0.40 & 0.16 & 0.01 & 0.03 \\
Folate $(\mu \mathrm{g})$ & 0.24 & 0.55 & 0.07 & -0.04 & 0 \\
Vitamin E $(\mathrm{mg})$ & 0.18 & 0.34 & 0.15 & 0.06 & -0.09 \\
Cholesterol $(\mathrm{mg})$ & 0.04 & 0.24 & 0.39 & 0.01 & -0.19 \\
\hline
\end{tabular}

RE, retinol equivalents.

Table 3 General characteristics of participants according to high blood pressure status. Korean adults $(n 5151)$ from the community-based KoGES cohort

\begin{tabular}{|c|c|c|c|c|c|c|c|}
\hline & \multicolumn{2}{|c|}{ High BP } & \multicolumn{2}{|c|}{ At risk } & \multirow[b]{2}{*}{$\mathrm{HR}$} & \multirow[b]{2}{*}{$95 \% \mathrm{Cl}$} & \multirow[b]{2}{*}{$P_{\text {trend }}$} \\
\hline & $n$ & $\%$ & $n$ & $\%$ & & & \\
\hline \multicolumn{8}{|l|}{ Age (years) } \\
\hline $40-49$ & 623 & $46 \cdot 6$ & 3098 & $60 \cdot 1$ & Ref. & - & \multirow[t]{4}{*}{$<0.001$} \\
\hline $50-59$ & 340 & $25 \cdot 5$ & 1174 & $22 \cdot 8$ & 1.26 & $1 \cdot 10,1 \cdot 46$ & \\
\hline 60-69 & 373 & $27 \cdot 9$ & 879 & $17 \cdot 1$ & 1.95 & $1 \cdot 66,2 \cdot 29$ & \\
\hline Female & 668 & $50 \cdot 0$ & 2735 & 53.1 & 0.65 & $0.57,0.76$ & \\
\hline \multicolumn{8}{|l|}{ Education } \\
\hline$<$ Elementary & 502 & 37.6 & 1405 & $27 \cdot 3$ & Ref. & - & \multirow[t]{4}{*}{$<0.001$} \\
\hline Middle school & 316 & 23.7 & 1209 & 23.5 & 0.90 & $0.77,1.05$ & \\
\hline High school & 376 & $28 \cdot 1$ & 1779 & 34.5 & 0.72 & $0.61,0.86$ & \\
\hline$\geq$ College & 134 & $10 \cdot 0$ & 734 & $14 \cdot 3$ & 0.58 & $0.46,0.73$ & \\
\hline \multicolumn{8}{|l|}{ Income (\$US/month) } \\
\hline$<1000$ & 535 & $40 \cdot 0$ & 1484 & $28 \cdot 8$ & Ref. & - & \multirow[t]{4}{*}{$<0.001$} \\
\hline 1000-1999 & 359 & $26 \cdot 9$ & 1546 & $30 \cdot 0$ & 0.72 & $0.62,0.83$ & \\
\hline 2000-3999 & 324 & $24 \cdot 3$ & 1612 & $31 \cdot 3$ & 0.72 & $0.61,0.85$ & \\
\hline$\geq 4000$ & 94 & $7 \cdot 0$ & 434 & 8.4 & 0.75 & $0.59,0.95$ & \\
\hline \multicolumn{8}{|l|}{ Occupation } \\
\hline Office worker & 97 & $7 \cdot 3$ & 463 & $9 \cdot 0$ & Ref. & - & \\
\hline Non-office worker & 884 & $66 \cdot 2$ & 3180 & $61 \cdot 7$ & 0.99 & $0.80,1.24$ & \\
\hline Housework & 351 & $26 \cdot 3$ & 1490 & 28.9 & 1.00 & $0.78,1.30$ & \\
\hline Married & 1199 & 89.8 & 4722 & 91.7 & 0.95 & $0.79,1.14$ & \\
\hline Family history of hypertension(yes) & 248 & $18 \cdot 6$ & 822 & $16 \cdot 0$ & 1.50 & $1.30,1.73$ & \\
\hline \multicolumn{8}{|l|}{ BMI $\left(\mathrm{kg} / \mathrm{m}^{2}\right)$} \\
\hline$<23$ & 384 & $28 \cdot 7$ & 1853 & $36 \cdot 0$ & Ref. & - & \multirow[t]{8}{*}{$<0.001$} \\
\hline $23-24.9$ & 346 & $25 \cdot 9$ & 1441 & $28 \cdot 0$ & 1.23 & $1.06,1.42$ & \\
\hline $25-29.9$ & 541 & 40.5 & 1685 & $32 \cdot 7$ & 1.51 & $1.30,1.76$ & \\
\hline$\geq 30$ & 64 & 4.8 & 171 & 3.3 & 1.64 & $1 \cdot 22,2 \cdot 20$ & \\
\hline Abdominal obesity* & 440 & 32.9 & 1126 & 21.9 & 1.54 & $1.33,1.77$ & \\
\hline Alcohol intake (current, yes) & 645 & $48 \cdot 3$ & 2505 & $48 \cdot 6$ & 1.06 & $0.94,1.21$ & \\
\hline Smoking status (current, yes) & 370 & $27 \cdot 7$ & 1346 & $26 \cdot 1$ & 1.08 & $0.90,1.30$ & \\
\hline Physical activity (yes) & 334 & $25 \cdot 0$ & 1448 & $28 \cdot 1$ & 0.93 & $0.82,1.06$ & \\
\hline \multicolumn{8}{|l|}{ Total energy intake (kJ) } \\
\hline$\leq 6389(\leq 1527 \mathrm{kcal})$ & 354 & $26 \cdot 5$ & 1287 & $25 \cdot 0$ & Ref. & - & \multirow[t]{4}{*}{0.076} \\
\hline $6393-7648$ (1528-1828 kcal) & 324 & $24 \cdot 3$ & 1288 & $25 \cdot 0$ & 1.01 & $0.87,1.18$ & \\
\hline 7653-9109 (1829-2177 kcal) & 311 & 23.3 & 1289 & $25 \cdot 0$ & $1 \cdot 10$ & $0.94,1.29$ & \\
\hline$\geq 9113(\geq 2178 \mathrm{kcal})$ & 347 & $26 \cdot 0$ & 1287 & $25 \cdot 0$ & $1 \cdot 14$ & $0.98,1.33$ & \\
\hline
\end{tabular}

KoGES, Korean Genome Epidemiology Study; BP, blood pressure; HR, hazard ratio; Ref., reference category.

Adjusted for age, gender, education, occupation, married, income, BMI, waist circumference and family history of hypertension.

${ }^{*}$ Criteria for abdominal obesity used waist circumference for males and females $\geq 90 \mathrm{~cm}$ and $\geq 85 \mathrm{~cm}$, respectively. 
Table 4 Cox proportional hazard rate ratios for high blood pressure according to quintile of dietary pattern by gender. Korean adults ( $n 5151)$ from the community-based KoGES cohort

\begin{tabular}{|c|c|c|c|c|c|c|c|c|c|c|c|c|c|c|c|c|c|c|c|c|}
\hline & \multicolumn{10}{|c|}{ Males (n2416) } & \multicolumn{10}{|c|}{ Females $(n 2735)$} \\
\hline & \multirow[b]{2}{*}{ Q1 } & \multicolumn{2}{|r|}{ Q2 } & \multicolumn{2}{|r|}{ Q3 } & \multicolumn{2}{|r|}{ Q4 } & \multicolumn{2}{|r|}{ Q5 } & \multirow[b]{2}{*}{$P_{\text {trend }}$} & \multirow[b]{2}{*}{ Q1 } & \multicolumn{2}{|r|}{ Q2 } & \multicolumn{2}{|r|}{ Q3 } & \multicolumn{2}{|r|}{ Q4 } & \multicolumn{2}{|r|}{ Q5 } & \multirow[b]{2}{*}{$P_{\text {trend }}$} \\
\hline & & $\mathrm{HR}$ & $95 \% \mathrm{Cl}$ & $\mathrm{HR}$ & $95 \% \mathrm{Cl}$ & $\mathrm{HR}$ & $95 \% \mathrm{Cl}$ & $\mathrm{HR}$ & $95 \% \mathrm{Cl}$ & & & $\mathrm{HR}$ & $95 \% \mathrm{Cl}$ & $\mathrm{HR}$ & $95 \% \mathrm{Cl}$ & $\mathrm{HR}$ & $95 \% \mathrm{Cl}$ & $\mathrm{HR}$ & $95 \% \mathrm{Cl}$ & \\
\hline \multicolumn{21}{|l|}{ ‘Fruit' pattern } \\
\hline No. of cases & 187 & 136 & & 142 & & 99 & & 104 & & & 80 & 112 & & 131 & & 165 & & 180 & & \\
\hline Model 1 & Ref. & 0.84 & $0.67,1.05$ & 1.08 & $0.87,1.35$ & 0.99 & $0.77,1.26$ & $1 \cdot 17$ & $0.92,1.49$ & 0.141 & Ref. & 1.09 & $0.82,1.46$ & 1.00 & $0.75,1.32$ & 1.07 & $0.82,1.39$ & 1.14 & $0.88,1.48$ & 0.371 \\
\hline Model 2 & Ref. & 0.88 & $0.70,1 \cdot 11$ & $1 \cdot 15$ & $0.91,1.46$ & 1.01 & $0.78,1.30$ & $1 \cdot 13$ & $0.89,1.45$ & 0.223 & Ref. & $1 \cdot 20$ & $0.89,1.62$ & 1.09 & $0.81,1.47$ & $1 \cdot 18$ & $0.88,1.57$ & $1 \cdot 13$ & $0.86,1.48$ & 0.628 \\
\hline \multicolumn{21}{|c|}{ 'Vegetable' pattern } \\
\hline Model 1 & Ref. & 1.07 & $0.86,1.33$ & $1 \cdot 17$ & $0.93,1.47$ & 1.07 & $0.85,1.36$ & $1 \cdot 13$ & $0.89,1.45$ & 0.314 & Ref. & 0.83 & $0.64,1.08$ & 0.85 & $0.66,1.09$ & 0.75 & $0.58,0.96$ & 1.03 & $0.81,1.30$ & 0.783 \\
\hline Model 2 & Ref. & 1.02 & $0.81,1.27$ & $1 \cdot 22$ & $0.97,1.54$ & 1.07 & $0.84,1.36$ & 1.09 & $0.85,1.40$ & 0.259 & Ref. & 0.90 & $0.68,1.17$ & 0.92 & $0.71,1.19$ & 0.84 & $0.64,1.09$ & $1 \cdot 16$ & $0.91,1.47$ & 0.344 \\
\hline \multicolumn{21}{|c|}{ ‘High-protein’ pattern } \\
\hline No. of cases & 114 & 125 & & 136 & & 120 & & 173 & & & 205 & 149 & & 120 & & 109 & & 85 & & \\
\hline Model 1 & Ref. & 0.89 & $0.69,1.15$ & 0.94 & $0.73,1.21$ & 0.77 & $0.59,1.00$ & 0.93 & $0.72,1.19$ & 0.393 & Ref. & 0.88 & $0.71,1.09$ & 0.86 & $0.68,1.08$ & 0.91 & $0.72,1.16$ & 0.99 & $0.77,1.29$ & 0.736 \\
\hline \multirow{2}{*}{\multicolumn{21}{|c|}{ ‘Coffee' pattern }} \\
\hline & & & & & & & & & & & & & & & & & & & & \\
\hline No. of cases & 102 & 124 & & 134 & & 121 & & 187 & & & 146 & 171 & & 152 & & 119 & & 80 & & \\
\hline Model 1 & Ref. & 1.38 & $1.06,1 \cdot 79$ & 1.44 & $1 \cdot 12,1 \cdot 87$ & $1 \cdot 18$ & $0.91,1.54$ & $1 \cdot 30$ & $1.02,1.65$ & 0.248 & Ref. & $1 \cdot 18$ & $0.95,1.47$ & $1 \cdot 25$ & $0.99,1.57$ & 0.94 & $0.74,1 \cdot 20$ & 1.07 & $0.81,1.41$ & 0.768 \\
\hline Model 2 & Ref. & 1.39 & $1.06,1.82$ & $1 \cdot 39$ & $1.07,1.80$ & $1 \cdot 21$ & $0.92,1.57$ & $1 \cdot 27$ & $1.00,1.62$ & 0.315 & Ref. & $1 \cdot 18$ & $0.94,1.49$ & $1 \cdot 25$ & $0.99,1.58$ & 0.95 & $0.74,1.22$ & $1 \cdot 10$ & $0.83,1.45$ & 0.894 \\
\hline Model 1 & Ref. & $1 \cdot 20$ & $0.90,1.62$ & 1.03 & $0.77,1.38$ & 1.34 & $1.02,1 \cdot 77$ & $1 \cdot 36$ & $1.04,1 \cdot 78$ & 0.013 & Ref. & 1.02 & $0.80,1.30$ & $1 \cdot 19$ & $0.93,1.52$ & 1.37 & $1.07,1.75$ & $1 \cdot 71$ & $1 \cdot 34,2 \cdot 19$ & $<0.001$ \\
\hline Model 2 & Ref. & 1.11 & $0.82,1.50$ & 1.03 & $0.76,1.39$ & 1.31 & $0.97,1.75$ & 1.22 & $0.92,1.62$ & 0.084 & Ref. & 0.94 & $0.73,1.22$ & 1.07 & $0.83,1.38$ & 1.13 & $0.87,1.46$ & 1.36 & $1.05,1.77$ & 0.004 \\
\hline
\end{tabular}

KoGES, Korean Genome Epidemiology Study; Q1, quintile 1; Q2, quintile 2; Q3, quintile 3; Q4, quintile 4; Q5, quintile 5; HR, hazard ratio; Ref., reference category.

Model 1, HR adjusted for age; Model 2, HR adjusted for age, education, income, family history of hypertension, BMI, waist circumference and total energy intake. 


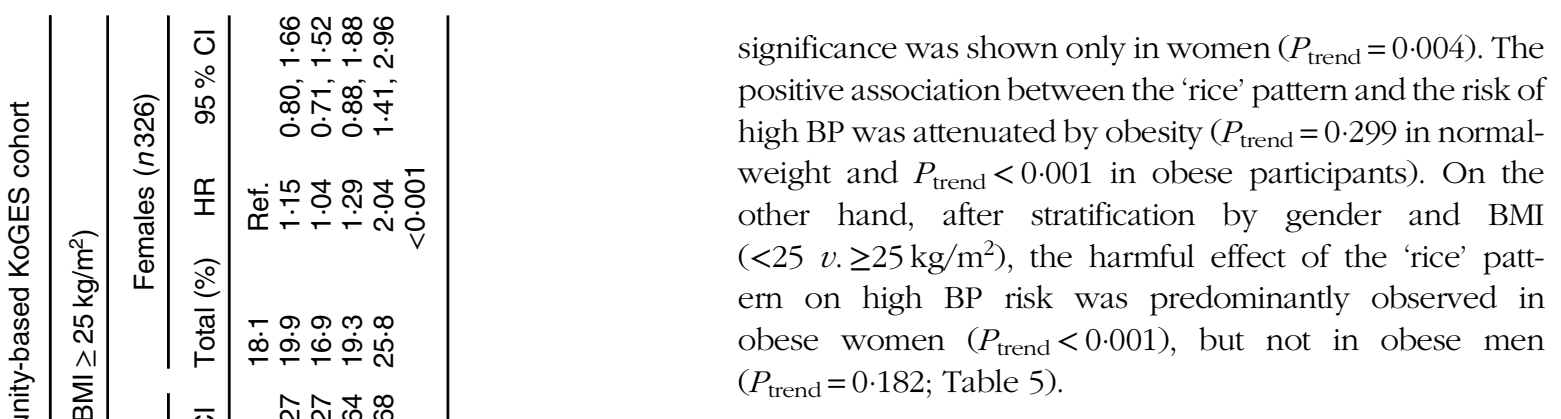

\section{Discussion}

The present community-based cohort study showed that the 'rice' pattern was positively associated with the risk of high BP. The 'rice' pattern was characterized by low scores of unrefined cereals in Korea but high scores of refined rice and various types of kimchi with high salt. After adjusting for all confounding factors, its statistical significance was shown only in women. The positive association was attenuated by obesity. On the other hand, the harmful effect of the 'rice' pattern on the risk of high BP was predominantly observed in obese women, but not in obese men, suggesting an attenuated effect of gender and obesity on the association.

The 'fruit' pattern scored higher in females, homemakers or unemployed persons, and those with abdominal obesity, but scored lower in current drinkers or current smokers compared with their counterparts in our study. Almost all ecological characteristics showed the opposite trend between the 'high-protein' pattern and 'rice' pattern except for gender and current smoking status. Individuals with high scores for the 'coffee' pattern were males, office workers and current smokers. Strong socio-economic gradients have been observed in dietary patterns identified in several studies, showing that higher incomes and education levels are usually associated with higher scores for healthy eating indices and healthy dietary patterns, but smoking, alcohol consumption and physical inactivity are related to unhealthy dietary patterns ${ }^{(24,25)}$.

In the present study, the effect of the 'rice' pattern on high BP risk could be considered according to two factors: (i) dietary $\mathrm{Na}$ intake and (ii) consumption of foods with high glycaemic index or high glycaemic load. First, the 2013 US Institute of Medicine report acknowledged the overall association between $\mathrm{Na}$ intake and $\mathrm{BP}$ based on previous studies ${ }^{(26)}$. Koreans consume 2-4-fold higher $\mathrm{Na}$ ( $4800 \mathrm{mg}$ ) than the daily intake recommended by $\mathrm{WHO}^{(27)}$. The top five foods contributing to $\mathrm{Na}$ intake are kimchi $(20.0 \%)$, salt $(17.5 \%)$, soya sauce $(8.7 \%)$, salt-fermented soya paste $(6.4 \%)$ and ramen $(4.4 \%)^{(28)}$; these foods scored highly in the 'rice' pattern in our study (see online supplementary material, Supplemental Table S1). A cross-sectional study (Korean National Health and Nutrition Examination Survey 2009-2011) recommended low salt intake for Koreans to prevent and 
control adverse $\mathrm{BP}^{(29)}$, and this is supported by our prospective study. The American Heart Association and the US Centers for Disease Control and Prevention have criticized the J- or U-shaped relationship between $\mathrm{Na}$ intake and hypertension risk $^{(30)}$, suggested that excessive salt intake is associated with hypertension. In the present study, it was suggested that the 'rice' pattern could increase $\mathrm{Na}$ intake and effect increased risk of high BP. Second, dietary carbohydrate intake has received considerable research attention because of a positive association of diets with high glycaemic index or high glycaemic load with risks of obesity, metabolic syndrome, diabetes and $\mathrm{CHD}^{(31)}$. A cross-sectional study ${ }^{(32)}$ suggested that the percentage of energy from carbohydrates and intake of refined grains are associated with Korean metabolic syndrome. Although several prospective studies worldwide have shown that dietary glycaemic index or glycaemic load is associated with high incidence of CHD, type 2 diabetes and stroke $^{(33,34)}$, to the best of our knowledge, no prospective study has reported the effect for Korean adults.

Due to ethnic variations, our results revealed that the 'rice' pattern rich in refined carbohydrates was positively associated with the risk of high BP in Korean adults, predominantly in women, consistent with the results of previous studies ${ }^{(15,33,35-37)}$. Gender differences in dietary practices among those who consumed high-carbohydrate diets showed differential effects on blood pressure in the present study, similar to the results by Song et al. ${ }^{(32)}$. The risk of metabolic disease might be related to female sex hormones, implying a presumably higher susceptibility of women than men to the risk of hypertension ${ }^{(20,38)}$. Pathophysiological mechanisms, the renin-angiotensin system, sex hormones and increased immune inflammatory factors might have greater impacts on women than on men ${ }^{(39,40)}$. Although there are many different pathophysiological and social reasons why such impacts might be greater for women, the gender-specific effect of the 'rice' pattern on high BP risk could suggest that reducing refined rice intake might be one approach to reduce the risk in women.

Obesity is not only a chronic condition, but also an important biological risk factor for non-communicable diseases. Diet has been widely identified as a factor in the prevention of obesity ${ }^{(21)}$. As an extended line of gender-specific effect, the risk of high BP for women with high score for the 'rice' pattern remained only for obese women, suggesting that the effect of the 'rice' pattern on high BP risk could be attenuated by obesity and gender. Obesity and insulin resistance are not caused by excessive dietary fat intake, but by carbohydrate intake that exceeds energy needs ${ }^{(41)}$. In the present study, increased TAG level was observed in participants with high scores for the 'rice' pattern (data not shown), and this is consistent with the results by Parks and Hellerstein ${ }^{(42)}$. The Nurses' Health Study has shown that the effect of carbohydrate intake on the risk of some components (TAG and HDL-cholesterol) of the metabolic syndrome is stronger in women with high BMI levels ${ }^{(43)}$.

The current study has notable strengths as it is the first prospective study to evaluate the effect of dietary pattern on the risk of high $\mathrm{BP}$ for Koreans who traditionally consume large amounts of rice as a staple food. To avoid latent period bias and evade a possible bias due to changes in lifestyle factors which could be caused by uncomfortable symptoms even without any diagnosis of high BP, our analysis was examined after excluding individuals who were diagnosed with high BP at baseline. Finally, we performed advanced analyses to evaluate the attenuated effect of gender and obesity on the association between dietary pattern and the risk of high BP.

Nevertheless, the study has several limitations. First, although assessment of our dietary patterns was based on indices that operationalized various (food) items and derived their information from validated FFQ, it had an inherent methodological limitation, due to self-report bias or recall bias, to reflect the general diet of our study population. Second, our FFQ might not have adequately covered changing dietary habits because dietary assessment was performed only once at baseline. Third, our study results cannot be generalized to other populations, especially populations with a low-carbohydrate staple diet, but could be considered in Asians who traditionally consume large amounts of high-carbohydrate food as their staple diet.

\section{Conclusion}

In conclusion, based on the present longitudinal study in Koreans, a positive association of the 'rice' pattern was found with long-term development of incident high BP, predominantly in women. In addition, the association is likely to be attenuated by gender and obese status; the harmful effect of the 'rice' pattern on the risk of high BP was not observed in normal-weight women but was only shown in obese women. These results could be viewed as discouraging in terms of the appropriate dietary modifications following the current guidelines and recommendations for the prevention of high BP.

\section{Acknowledgements}

Financial support: This work was supported by a 2015 Research Grant from Kangwon National University (number 520150345). The funding source was not involved in the design, implementation, analyses or interpretation of the data. Conflict of interest: The authors declare no conflict of interest. Authorship: H.Y., D.K. and S.-A.L. designed and conducted the research; H.Y. and S.A.-L. analysed the data and performed the statistical analyses; H.Y. and S.-A.L. 
wrote the manuscript and had primary responsibility for the final content of the manuscript; and all authors read and approved the final manuscript. Ethics of buman subject participation: This study was conducted according to the guidelines laid down in the Declaration of Helsinki and all procedures involving human subjects were approved by the Institutional Review Board of Kangwon National University Hospital. Written informed consent was obtained from all subjects.

\section{Supplementary material}

To view supplementary material for this article, please visit https://doi.org/10.1017/S136898001900168X

Author ORCID. (1) Sang-Ah Lee, 0000-0002-5079-9733.

\section{References}

1. World Health Organization (2014) Global Status Report on Noncommunicable Diseases 2014. Geneva: WHO.

2. Lewington S, Clarke R, Qizilbash N et al. (2002) Age-specific relevance of usual blood pressure to vascular mortality: a meta-analysis of individual data for one million adults in 61 prospective studies. Lancet 360, 1903-1913.

3. Chobanian AV, Bakris GL, Black HR et al. (2003) The seventh report of the Joint National Committee on Prevention, Detection, Evaluation, and Treatment of High Blood Pressure: the JNC 7 report. JAMA 289, 2560-2572.

4. Li B, Li F, Wang L et al. (2016) Fruit and vegetables consumption and risk of hypertension: a meta-analysis. J Clin Hypertens 18, 468-476.

5. Ralston RA, Lee JH, Truby H et al. (2012) A systematic review and meta-analysis of elevated blood pressure and consumption of dairy foods. J Hum Hypertens 26, 3-13.

6. Chan Q, Stamler J, Griep LM et al. (2016) An update on nutrients and blood pressure. J Atheroscler Thromb 23 276-289.

7. Aljefree N \& Ahmed F (2015) Association between dietary pattern and risk of cardiovascular disease among adults in the Middle East and North Africa region: a systematic review. Food Nutr Res 59, 27486.

8. Lee SA, Cai H, Yang G et al. (2010) Dietary patterns and blood pressure among middle-aged and elderly Chinese men in Shanghai. Br J Nutr 104, 265-275.

9. Niu K, Momma H, Kobayashi Y et al. (2016) The traditional Japanese dietary pattern and longitudinal changes in cardiovascular disease risk factors in apparently healthy Japanese adults. Eur J Nutr 55, 267-279.

10. Park JE, Jung H \& Lee JE (2014) Dietary pattern and hypertension in Korean adults. Public Health Nutr 17, 597-606.

11. Sadakane A, Tsutsumi A, Gotoh T et al. (2008) Dietary patterns and levels of blood pressure and serum lipids in a Japanese population. J Epidemiol 18, 58-67.

12. Shin JY, Kim JM \& Kim Y (2013) Associations between dietary patterns and hypertension among Korean adults: the Korean National Health and Nutrition Examination Survey (20082010). Nutr Res Pract 7, 224-232.

13. Wang D, He Y, Li Y et al. (2011) Dietary patterns and hypertension among Chinese adults: a nationally representative cross-sectional study. BMC Public Health 11, 925.
14. Xu X, Byles J, Shi Z et al. (2016) Dietary pattern transitions, and the associations with BMI, waist circumference, weight and hypertension in a 7-year follow-up among the older Chinese population: a longitudinal study. BMC Public Health 16, 743.

15. Kim K, Yun SH, Choi BY et al. (2008) Cross-sectional relationship between dietary carbohydrate, glycaemic index, glycaemic load and risk of the metabolic syndrome in a Korean population. Br J Nutr 100, 576-584.

16. Radhika G, Van Dam RM, Sudha V et al. (2009) Refined grain consumption and the metabolic syndrome in urban Asian Indians (Chennai Urban Rural Epidemiology Study 57). Metabolism 58, 675-681.

17. Hooper L, Martin N, Abdelhamid A et al. (2015) Reduction in saturated fat intake for cardiovascular disease. Cochrane Database Syst Rev issue 6, CD011737.

18. Mozaffarian D, Micha R \& Wallace S (2010) Effects on coronary heart disease of increasing polyunsaturated fat in place of saturated fat: a systematic review and meta-analysis of randomized controlled trials. PLoS Med 7, e1000252.

19. Li Y, Hruby A, Bernstein AM et al. (2015) Saturated fats compared with unsaturated fats and sources of carbohydrates in relation to risk of coronary heart disease: a prospective cohort study. J Am Coll Cardiol 66, 1538-1548.

20. Mosher MJ, Martin LJ, Cupples LA et al. (2005) Genotype-bysex interaction in the regulation of high-density lipoprotein: the Framingham Heart Study. Hum Biol 77, 773-793.

21. World Health Organization (2003) Diet, Nutrition and the Prevention of Chronic Diseases. Report of a Joint WHO/ FAO Expert Consultation. WHO Technical Report Series no. 916. Geneva: WHO.

22. Lim S, Jang HC, Lee HK et al. (2006) A rural-urban comparison of the characteristics of the metabolic syndrome by gender in Korea: the Korean Health and Genome Study (KHGS). J Endocrinol Invest 29, 313-319.

23. Ahn Y, Kwon E, Shim JE et al. (2007) Validation and reproducibility of food frequency questionnaire for Korean genome epidemiologic study. EurJ Clin Nutr 61, 1435-1441.

24. Heroux M, Janssen I, Lam M et al. (2010) Dietary patterns and the risk of mortality: impact of cardiorespiratory fitness. Int $J$ Epidemiol 39, 197-209.

25. Mullie P, Clarys P, Hulens M et al. (2010) Dietary patterns and socioeconomic position. Eur J Clin Nutr 64, 231-238.

26. Graudal N (2015) Dietary sodium: where science and policy conflict: impact of the 2013 IOM Report on Sodium Intake in Populations. Curr Hypertens Rep 17, 9.

27. Korea Centers for Disease Control and Prevention (2011) Korea Health Statistics 2011: Korea National Health and Nutrition Examination Survey (KNHANES V-2). Osong: Korea Centers for Disease Control and Prevention.

28. Korea Centers for Disease Control and Prevention (2009) Korean National Health Statistics - KNHANES 2008. Osong: Korea Centers for Disease Control and Prevention.

29. Oh J, Lee J, Koo HS et al. (2014) Estimated 24-hour urine sodium excretion is correlated with blood pressure in Korean population: 2009-2011 Korean National Health and Nutritional Examination Survey. J Korean Med Sci 29, Suppl. 2, S109-S116.

30. Cogswell ME, Mugavero K, Bowman BA et al. (2016) Dietary sodium and cardiovascular disease risk - measurement matters. $N$ Engl J Med 375, 580-586.

31. Barclay AW, Petocz P, McMillan-Price J et al. (2008) Glycemic index, glycemic load, and chronic disease risk - a meta-analysis of observational studies. Am J Clin Nutr 87, 627-637.

32. Song S, Lee JE, Song WO et al. (2014) Carbohydrate intake and refined-grain consumption are associated with metabolic syndrome in the Korean adult population. J Acad Nutr Diet 114, 54-62.

33. Liu S, Willett WC, Stampfer MJ et al. (2000) A prospective study of dietary glycemic load, carbohydrate intake, and risk 
of coronary heart disease in US women. Am J Clin Nutr $\mathbf{7 1}$, 1455-1461.

34. Oba S, Nagata C, Nakamura K et al. (2010) Dietary glycemic index, glycemic load, and intake of carbohydrate and rice in relation to risk of mortality from stroke and its subtypes in Japanese men and women. Metabolism 59, 1574-1582.

35. Choi J, Se-Young O, Lee D et al. (2012) Characteristics of diet patterns in metabolically obese, normal weight adults (Korean National Health and Nutrition Examination Survey III, 2005). Nutr Metab Cardiovasc Dis 22, 567-574.

36. Nanri A, Mizoue T, Noda M et al. (2010) Rice intake and type 2 diabetes in Japanese men and women: the Japan Public Health Center-based Prospective Study. Am J Clin Nutr 92 , 1468-1477.

37. Park SH, Lee KS, Park HY (2010) Dietary carbohydrate intake is associated with cardiovascular disease risk in Korean: analysis of the third Korea National Health and Nutrition Examination Survey (KNHANES III). Int J Cardiol 139, 234-240.
38. Mittendorfer B (2005) Insulin resistance: sex matters. Curr Opin Clin Nutr Metab Care 8, 367-372.

39. Balan H \& Popescu L (2014) 'Gender specific medicine': a focus on gender-differences in hypertension. Rom $J$ Intern Med 52, 129-141.

40. Pechere-Bertschi A \& Burnier M (2004) Female sex hormones, salt, and blood pressure regulation. $\mathrm{Am} \mathrm{J}$ Hypertens 17, 994-1001.

41. Cheung BM (2005) The cardiovascular continuum in Asia - a new paradigm for the metabolic syndrome. I Cardiovasc Pharmacol 46, 125-129.

42. Parks EJ \& Hellerstein MK (2000) Carbohydrate-induced hypertriacylglycerolemia: historical perspective and review of biological mechanisms. Am J Clin Nutr 71, 412-433.

43. Liu S, Manson JE, Stampfer MJ et al. (2001) Dietary glycemic load assessed by food-frequency questionnaire in relation to plasma high-density-lipoprotein cholesterol and fasting plasma triacylglycerols in postmenopausal women. Am J Clin Nutr 73, 560-566. 